\title{
A Multi-Step Procedure for Enriching Limited Two-Dimensional Acoustic Far-Field Pattern Measurements
}

\author{
Hélène Barucd*, Chokri Bekkeyt, Rabia Djelloulit \\ Thème : Observation et modélisation pour les sciences de l'environnement \\ Équipe-Projet Magique 3D
}

Rapport de recherche $\mathrm{n}^{\circ} 7048$ - Octobre 2009 - 30 pages

\begin{abstract}
We propose a three-step solution methodology to increase the discrete set of acoustic far-field pattern (FFP) measurements, available in a small range of observation angles (small aperture). The first two steps of the proposed procedure allow the extension of the data to an aperture larger than $\pi / 2$. They use a regularized Newton algorithm where the total variation of the FFP is incorporated as a regularization term. The third step consists in applying the standard Tikhonov regularization technique to recover the full aperture of the FFP from the previously extended field. Numerical results obtained using synthetic data illustrate the potential of the proposed procedure for reconstructing the full aperture of the FFP from data given in an aperture as small as backscattering measurements.
\end{abstract}

Key-words: acoustic scattering problem, limited aperture, inverse obstacle problem, ill-posed problem, total variation, Tikhonov regularization, Newton method.

\footnotetext{
* INRIA Bordeaux Sud-Ouest Research Center, Team Project Magique-3D, \& LMA/CNRS UMR 5142, Université de Pau et des Pays de l'Adour, France

${ }^{\dagger}$ Faculté des Sciences de Monastir, Tunisia

¥ Department of Mathematics, California State University Northridge \& INRIA Bordeaux Sud-Ouest Research Center, Associate Team Project MAGIC, USA
}

Centre de recherche INRIA Bordeaux - Sud Ouest

Domaine Universitaire - 351, cours de la Libération 33405 Talence Cedex

Téléphone : +33540006900 


\section{Une méthode itérative pour l'extrapolation de l'amplitude de diffusion du champ acoustique 2D}

Résumé : Nous proposons une stratégie d'extrapolation de l'amplitude de diffusion du champ acoustique à partir de données mesurées sur un petit secteur d'observation. La procédure de reconstruction du champ est de type itératif. Elle s'appuie sur trois étapes et utilise la variation totale de l'amplitude de diffusion à des fins de régularisation. Les résultats numériques obtenus à partir de mesures synthétiques illustrent l'efficacité de la méthode proposée pour reconstruire entièrement $\left(\right.$ sur $\left.360^{\circ}\right)$ l'amplitude de diffusion et cela à partir de très peu de points de mesure ( même un seul point) et pour différents niveaux de bruits.

Mots-clés : problème de diffraction acoustique, problème inverse, problème mal posè, fenêtre réduite d'observation, variation totale, régularisation de Tikhonov, mèthode de Newton 


\section{Introduction}

The development of efficient solution methodologies for solving inverse problems is very important to many technologies such as sonar, radar, geophysical exploration, medical imaging and nondestructive testing [1]. A typical example of inverse problems is the determination of the shape of an obstacle from the knowledge of some scattered far-field patterns (FFP), and assuming some a priori knowledge about the characteristics of the surface of the obstacle. This inverse obstacle problem (IOP) is very difficult to solve numerically because it is not only nonlinear [2], but most importantly improperly posed in the sense of Hadamard [3]. Because of its practical importance, the IOP has received a great deal of attention by applied mathematicians and engineers during the last three decades leading to the design of various computational methods (see for example the overview in [4] and references therein). The numerical results reported in the literature indicate that the success in the reconstruction of the sought-after shape of an obstacle by the current numerical methods depends strongly on the quality of the given FFP measurements: the aperture (range of observation angles) and the level of noise in the data (accuracy of measurements). More specifically, it has been observed that it is possible to retrieve a unique and reasonably good solution of the IOP when using only one incident plane wave (one frequency and one incident direction) and measured FFP around the entire obstacle (full-aperture problem ), even when the measurements are contaminated with a high level of white noise (up to 20\%) [5]. The situation is quite different in the case of the limited aperture problem [6, 7, 8, 9] which is of great importance and interest since in most applications the FFP cannot be measured entirely but only in a limited sector. Indeed, early numerical results (see for example [6, 10, 11, 12] among others) performed in the resonance region - that is, for a wavelength that is approximately equals to the diameter of the obstacle — showed that using anywhere from 13 to 24 incident waves and limited-aperture far-field data and as long as the aperture is larger than $\pi$, one can achieve a good reconstruction of the shape of the obstacle. For smaller apertures, the reconstruction becomes more difficult and nearly impossible for apertures smaller than $\pi / 4$, even when the measurements are noise free. Later numerical results [14] demonstrated that it is -in fact-possible to obtain a reasonable reconstruction of the shape of an obstacle from the knowledge of its corresponding FFP when measured in an aperture of $\pi / 2$ for one incident plane wave only. The quality of the reconstruction can be significantly improved depending on the number of incident plane waves (multiple incident directions and/or frequencies) as well as on the noise level [14].

Given that, it is of paramount to develop numerical procedures to enrich (increase the size) the set of FFP measurements when given in a small aperture. Note that a procedure for denoising the FFP data while solving simultaneously the IOP has been recently proposed in [15]. However, as it has been stated earlier, there is no hope -at least with existing numerical methods- to solve the IOP when the FFP data are given in a small apertures (less than $\pi / 4$ ) even when the data are noise free. Consequently, we propose a three-step solution methodology to extend the range of FFP data when measured in a small range of observation angles (small aperture). The goal of the procedure is to extend the few measured FFP data to -at least- a range of $\pi / 2$ in order to be used for solving efficiently the IOP problem. From a mathematical point of view, it is always possible to extend the FFP uniquely to the entire circle $S$ when given in a (continuous) subset of $S$. This unique determination is due to the analyticity of the FFP [2]. However, the numerical extension from the knowledge of a (discrete) subset 
of the FFP is a very challenging problem. Indeed, such extension can be formulated as an inverse problem that is extremely ill-posed due precisely to the analyticity of the FFP. Therefore, any numerical procedure for extending (enriching) the FFP measurements must address efficiently the ill-posedness nature of this inverse problem. Previous attempts to solve this problem were based on using the standard $L^{2}$-Tikhonov regularization technique [13 14]. The extension was (to some extent) successful only when the range of measurements is given in an aperture larger than $\pi / 2$. The procedure fails to address situations of practical interest, that is when only one measurement (backscattering) or few measurements are available. The computational method we propose, in its first two steps, extends the data to an aperture larger than $\pi / 2$. The third step allows to extend of the FFP to its full aperture. The first two steps of the proposed procedure employ a regularized Newton-type algorithm where the total variation (TV) of the FFP is incorporated to restore the stability to the inverse problem. The idea for using the TV formulation is not new [26]. It has already been sucessfully used in image deblurring applications [27]-[30]. However, it has not been employed by applied mathematicians and engineers to other applications since it leads to the minimization of a non-differentiable cost function. The lack of differentiability prevents using robust minimization algorithms (such as Newton algorithm) involving the computation of jacobians. We employ a modified version of this regularization technique that leads to the minimization of a differentiable cost function. The third step of the proposed procedure uses a Tikhonov-type regularization technique that is known to be efficient when the data are given on an aperture larger than $\pi / 2$. The numerical results reported in this paper illustrate the efficiency of the proposed solution methodology for reconstructing the FFP to full aperture in the low frequency regime from noisy synthetic measurements as few as backscattering data.

The remainder of this paper is organized as follows. In Section 2, we specify the notations and assumptions adopted in this paper, and we recall the direct and inverse acoustic scattering problems. Section 3 is devoted to the description of the proposed multi-step solution methodology for enriching FFP data. We introduce the mathematical formulation of the inverse FFP problem (IFP). This problem is then regularized using the total variation of the FFP. Since the cost-function corresponding to this regularized IFP problem is not differentiable, a modified version of this regularization is adopted in order to restore the regularity. Then, we describe the algorithm of the solution procedure and summarize its computational complexity. Section 4 is devoted to the numerical results obtained using synthetic data. These results illustrate the salient features and demonstrate the performance characteristics of the proposed solution methodology. We state our conclusion in Section 5. Appendix A contains auxiliary properties pertaining to the TV formulation.

\section{Preliminaries}

We specify in this section the nomenclature and assumptions that are adopted throughout this paper. 


\subsection{The Direct Acoustic Scattering Problem}

We consider throughout this paper the class of acoustic scattering problems in the case of two-dimensional sound-soft scatterers. It consists in finding the scattered field $u$, solution of the following boundary value problem (BVP) [2]:

$$
(\mathrm{BVP}) \begin{cases}\Delta u+k^{2} u=0 & \text { in } \Omega^{e} \\ u=-u^{\mathrm{inc}} & \text { on } \Gamma \\ \lim _{\|\boldsymbol{x}\|_{2} \rightarrow \infty} \sqrt{\|\boldsymbol{x}\|_{2}}\left(\frac{\partial u}{\partial\|\boldsymbol{x}\|_{2}}-\mathrm{i} k u\right)=0 & \end{cases}
$$

where $u^{\text {inc }}$ is the incident plane wave given by

$$
u^{\mathrm{inc}}=e^{\mathrm{i} k \boldsymbol{x} \cdot \boldsymbol{d}}
$$

and $\Omega^{e}$ is a homogeneous unbounded domain surrounding the sound-soft scatterer $\Omega$, that is $\Omega^{e}=\mathbb{R}^{2} \backslash \bar{\Omega}, \Gamma$ is the boundary of the scatterer, $k$ is a positive number representing the wavenumber, and $\boldsymbol{d}$ is a unit vector representing the direction of the incident plane wave. Note that the couple $(k, \boldsymbol{d})$ characterizes the incident plane wave $u^{\text {inc }}$. The last equation of BVP is called the Sommerfeld radiation condition. We point out that the Dirichlet boundary condition is used only for simplicity and that the results presented herein apply to Neumann and Robin-type boundary conditions, that are usually encountered in acoustic scattering problems.

The far-field pattern $u_{\infty}$ of the solution $u$ of BVP is defined on the unit circle $S$ from the asymptotic behavior of the scattered field $u$ (see, for example, [2]),

$$
u(\boldsymbol{x})=\frac{e^{i k r}}{\sqrt{r}}\left(u_{\infty}(\theta)+O\left(\frac{1}{r}\right)\right), \quad r \longrightarrow+\infty
$$

where $(r, \theta)$ are the polar coordinates corresponding to the cartesian coordinates $\boldsymbol{x}$; $r>0$ and $\theta \in[0,2 \pi)$. Note that the direct acoustic scattering problem BVP has been extensively investigated mathematically, and a considerable amount of results pertaining to the existence, uniqueness, regularity, and asymptotic behavior of the solution $u$ can be found in [2], [16]-[19], among others. The reader interested in the solution methodologies for solving BVP is invited to read, for example, the recent monograph [20].

\subsection{The Inverse Obstacle Problem}

First, we note that the solution $u$ of BVP defines an operator $F: \Gamma \rightarrow u_{\infty}$ which maps the boundary $\Gamma$ of the scatterer $\Omega$ onto the far-field pattern $u_{\infty}$. Hence, inverse obstacle problems (IOP) can be formulated as follows:

Given one or several measured far-field patterns $\tilde{u}_{\infty}(\hat{\theta})$, corresponding to one or several given directions $\boldsymbol{d}$ and wavenumbers $k$, find $\Gamma$ that satisfies:

$$
F(\Gamma)(\hat{\theta})=\widetilde{u}_{\infty}(\hat{\theta}) ; \quad \hat{\theta} \in S
$$

where the tilde notation designates a measured quantity.

The following three observations are noteworthy 
- Although IOP is one of the simplest problems arising in the inverse scattering field, it is still not completely solved from a mathematical view point. Indeed, only partial results pertaining to the uniqueness of the solution of IOP are available [2] 18 21]. On the other hand, numerous solution methodologies have been proposed during the last three decades to solve numerically IOP (see, for example, the overview in [4] and the references therein).

- The FFP measurements $\widetilde{u}_{\infty}(\hat{\theta})$ in the IOP problems are not available for all $\hat{\theta} \in$ $S$ (full-aperture). Indeed, for most applications, it is possible to measure $\widetilde{u}_{\infty}(\hat{\theta})$ in only an open subset of $S$ (limited-aperture). Typically, $\widetilde{u}_{\infty}(\hat{\theta})$ is given at some observation points $\hat{\theta}_{j} ; 1 \leq j \leq M$ for multiple incident directions and/or frequencies. The important case of backscattering measurements corresponds to $M=1$.

- It is well known that the success for solving numerically IOP depends on the quality of the FFP data, that is the number of measurements, their location around the sought-after obstacle, and the noise level in the measurements.

\section{The Multi-Step Procedure for Enriching the FFP data}

This section is devoted to the description of the solution methodology we propose to extend the FFP data to full aperture when given in a limited observation sector. It is organized as follows. First, we formulate the FFP extension problem as an inverse problem. Then, we introduce the regularized iterative algorithm for solving this problem. Last, we describe the iterative extension process of the data. A summary of the numerical procedure concludes this section.

As stated earlier in the introduction, the idea for extending the measured FFP in a limited aperture to a wider range before solving the IOP problem is not new [13, 14] . A standard $L^{2}$-Tikhonov regularization technique was used for this purpose. The extension was successful only when the range of measurements is given in an aperture larger than $\pi / 2$. The procedure fails to address situations of practical interest where backscattering or only few noisy measurements are available.

We recall that it is possible to solve IOP, using regularized Newton-type methods, when FFP data are measured in (a) an aperture larger or equal to $\pi$, for one incident plane wave (one incident direction and one frequency), and (b) an aperture as small as $\pi / 2$ but for multiple incident plane waves (several incident directions and/or frequencies) [6], [10, 11] [12]. Given that, the proposed multi-step solution procedure must be able to extend the FFP data to a range of -at least $-\pi / 2$, when given in an aperture as small as backscattering measurements, in order to be of practical interest.

\subsection{Problem Statement}

Since the FFP $u_{\infty}$ corresponding to the solution $u$ of BVP is analytic on the unit circle $S$, we can express it as a Fourier series as follows:

$$
u_{\infty}(\theta)=\sum_{n=-\infty}^{\infty}(-i)^{n} c_{n} e^{i n \theta} ; \quad \theta \in S
$$

where the complex constants $c_{n}$ are the Fourier coefficients. The determination of these constants allows to measure the FFP $u_{\infty}$ on the entire unit circle $S$. 
The computation of the Fourier coefficients $c_{n}$ when the FFP $u_{\infty}$ is given at some (few) observation points can be formulated as the following inverse Fourier coefficients problem (IFP):

Given a set of $M$ far-field pattern measurements $\widetilde{\mathbf{u}}_{\infty}=\left[\widetilde{u}_{\infty}\left(\hat{\theta}_{1}\right), \cdots, \widetilde{u}_{\infty}\left(\hat{\theta}_{M}\right)\right]^{T}$ for one incident plane wave, find

$$
\hat{\mathbf{c}}=\arg \min _{\mathbf{c} \in \mathbb{C}^{2 N+1}}\left\|\mathbf{A c}-\widetilde{\mathbf{u}}_{\infty}\right\|_{2}
$$

where $\arg$ is used to denote that $\mathbf{c}$ is the minimizer of the cost function $\left\|\mathbf{A c}-\widetilde{\mathbf{u}}_{\infty}\right\|_{2}$ over $\mathbb{C}^{2 N+1}$ with $N$ being the truncating order of the Fourier series given by Eq. (3). $A$ is a $M \times(2 N+1)$ matrix given by:

$$
\mathbf{A}=\left[\begin{array}{ccccc}
(-\mathrm{i})^{-N} e^{-\mathrm{i} N \hat{\theta}_{1}} & \cdots & 1 & \cdots & (-\mathrm{i})^{N} e^{\mathrm{i} N \hat{\theta}_{1}} \\
(-\mathrm{i})^{-N} e^{-\mathrm{i} N \hat{\theta}_{2}} & \cdots & 1 & \cdots & (-\mathrm{i})^{N} e^{\mathrm{i} N \hat{\theta}_{2}} \\
\vdots & \vdots & \vdots & \vdots & \vdots \\
(-\mathrm{i})^{-N} e^{-\mathrm{i} N \hat{\theta}_{M}} & \cdots & 1 & \cdots & (-\mathrm{i})^{N} e^{\mathrm{i} N \hat{\theta}_{M}}
\end{array}\right]
$$

The IFP problem given by Eq. (4) is severely ill-posed. The condition number of the matrix $A$ given by Eq. (5) increases exponentially as the number of terms $N$ increases [2]. Consequently, it is very difficult to solve numerically IFP since the accuracy of the solution requires $N$ to be relatively large, depending on the frequency. For this reason, a stabilization technique must be incorporated during the solution of Eq. (4).

\subsection{The total variation regularization technique: A modified pro- cedure}

As stated in the previous paragraph, the numerical solution of the IFP problem requires first to restore the stability to Eq. (4). Stabilization procedures such as Tikhonov's regularization [22] 23] are the primary candidates for this class of problems. Such techniques consist in incorporating a 2-norm penalty-like term in Eq. (4) to make the resulting system better conditioned. Therefore, the minimization problem is replaced by the following regularized IFP problem:

$$
\hat{\mathbf{c}}=\arg \min _{\mathbf{c} \in \mathbb{C}^{2 N+1}}\left\{\frac{1}{2}\left\|\mathbf{A c}-\widetilde{\mathbf{u}}_{\infty}\right\|_{2}^{2}+\mu\|\mathbf{T}(\mathbf{c})\|_{2}^{2}\right\}
$$

where $\mu>0$ is the regularization parameter and $\mathbf{T}$ is a regularization operator. The simplest choice of the operator $\mathbf{T}$ is the identity mapping [13] corresponding to the standard Tikhonov regularization technique. $\mathbf{T}$ can also be either the first- or secondorder derivative operator [14]. The latter approach can be interpreted as a Tikhonov regularization technique which balances a goodness-of-fit measure and a roughness penalty. The regularization parameter $\mu$ can be expected to play the role of a smoother because it controls in this case the trade-off between goodness-of-fit and roughness. This parameter can be determined by theoretical considerations such as Morozov's discrepancy principle [24 25]. However, in both approaches the "optimal" value of the 
regularization parameter $\mu$ was found simply with a trial and error procedure, as it is often the case for most of regularized procedures. Last, both regularization strategies exhibit poor performance as soon as the range of observation angles (aperture) is less than $\pi / 2$, even in the case of noise free data. We must point out that we have observed that in the case of the standard regularization ( $T$ being the identity operator), the performance deteriorates when the FFP measurements (even when given in an aperture of $\pi$ ) are tainted with only $1 \%$ of white noise.

Given that, we propose to use the total variation (TV) of the far-field pattern to restore the stability to the IFP problem given by Eq. (4) [26]. Such technique has been used widely and successfully in image deblurring applications [27]-[30]. It consists in replacing the minimization problem (4) by the following TV-regularized IFP problem:

$$
\hat{\mathbf{c}}=\arg \min _{\mathbf{c} \in \mathbb{C}^{2 N+1}}\left\{\frac{1}{2}\left\|\mathbf{A c}-\widetilde{\mathbf{u}}_{\infty}\right\|_{2}^{2}+\mu\|\mathbf{D} \mathbf{c}\|_{1}\right\}
$$

where $\mathbf{D}$ is a $(2 N+1) \times(2 N+1)$ matrix representing the central discrete first-order derivative operator (see appendix). $\mathbf{D}$ is given by:

$$
\mathbf{D}=\frac{1}{2 h}\left[\begin{array}{cccccc}
-2 & 2 & 0 & 0 & \cdots & 0 \\
-1 & 0 & 1 & 0 & \cdots & 0 \\
\vdots & \vdots & \vdots & \vdots & \vdots & \vdots \\
0 & \cdots & 0 & -1 & 0 & 1 \\
0 & \cdots & 0 & 0 & -2 & 2
\end{array}\right]
$$

and $h$ is a positive parameter that results from the discrete approximation of the total variation (see appendix A).

Observe that this regularized formulation incurs a non-differentiable cost function due to the presence of the 1-norm term $\|\mathbf{D c}\|_{1}$. The lack of the regularity is a serious defect in the cost function since it rules out the use of efficient numerical procedures, such as Newton algorithms, that require the evaluation of the jacobians. Consequently, we modify the TV formulation, using a perturbation-type procedure suggested in [30], to restore the regularity. More specifically, we replace Eq. (7) by the following minimization problem:

$$
\hat{\mathbf{c}}=\arg \min _{\mathbf{c} \in \mathbb{C}^{2 N+1}}\left\{\frac{1}{2}\left\|\mathbf{A c}-\widetilde{\mathbf{u}}_{\infty}\right\|_{2}^{2}+\mu\left\|\left((\mathbf{D c})^{\mathbf{2}}+\beta^{2} \widetilde{\mathbf{e}}\right)^{\mathbf{1 / 2}}\right\|_{1}\right\}
$$

where $\widetilde{\mathbf{e}}$ is a vector in $\mathbb{C}^{2 N+1}$ introduced to restore the regularity to the $L^{1}$ norm term. $\widetilde{\mathbf{e}}$ is given by:

$$
\widetilde{\mathbf{e}}=[1, \cdots, 1]^{T}
$$

The bold face exponents in Eq. (8), and for the remaining of this section, are pointwise operations. The positive constant $\beta$ is the regularity parameter. Indeed, the presence of $\beta$ ( $\beta$ large enough) ensures the differentiability of the $L^{1}$-norm term in the cost function given by Eq. (8). The optimal value of the couple $(\mu, \beta)$ is obtained using - at this point of the study-a trial and error strategy since our primary goal is to investigate the feasibility of the proposed method. Note that there is no need for computing the positive parameter $h$ since, in practice, it is "absorbed" into the parameters $\mu$ and $\beta$. 
We note that a serious shortcoming of the standard $L^{2}$ regularization techniques is that they do not allow discontinuous solutions, whereas a TV-computed solution could be discontinuous. Therefore, the proposed TV formulation has the potential to be more robust in the presence of noisy data due to measurement errors and/or roundoff.

\subsection{The Newton Algorithm}

We propose to apply the Newton method to solve the nonlinear minimization problem given by since the corresponding cost function is now differentiable. Consequently, at each iteration $\mathbf{m}$, we solve the linear system:

$$
\mathcal{F}^{\prime \prime}\left(\mathbf{c}^{(\mathbf{m})}\right) \delta \mathbf{c}^{(\mathbf{m})}=-\mathcal{F}^{\prime}\left(\mathbf{c}^{(\mathbf{m})}\right)
$$

and then update

$$
\mathbf{c}^{(\mathbf{m}+\mathbf{1})}=\mathbf{c}^{(\mathbf{m})}+\delta \mathbf{c}^{(\mathbf{m})}
$$

The matrix $\mathcal{F}^{\prime \prime}$ is the Hessian of the regularized cost function given by $8 \mathcal{F}^{\prime \prime}$ is a $(2 N+1) \times(2 N+1)$ matrix given by [30]:

$$
\mathcal{F}^{\prime \prime}\left(\mathbf{c}^{(\mathbf{m})}\right)=\mathbf{A}^{*} \mathbf{A}+\mu\left(\mathbf{D}^{*} \Psi^{-1} \mathbf{D}-\mathbf{D}^{*}\left[\left(\mathbf{D} \mathbf{c}^{(\mathbf{m})}\right)\right]^{2} \Psi^{-3} \mathbf{D}\right)
$$

where $(\mathbf{c})$ is a $(2 N+1) \times(2 N+1)$ diagonal matrix whose diagonal entries are the components of the vector $\mathbf{c} \in \mathbb{C}^{2 N+1}$. $\Psi$ is a $(2 N+1) \times(2 N+1)$ diagonal matrix given by [30]:

$$
\Psi=\left(\left[\left[\mathrm{Dc} \mathbf{c}^{(\mathrm{m})}\right]^{2}+\beta^{2} \widetilde{\mathbf{e}}\right]^{1 / 2}\right)
$$

The vector $\mathcal{F}^{\prime}$ is the jacobian of the regularized cost function given by $\left[8 . \mathcal{F}^{\prime}\left(\mathbf{c}^{(\mathbf{m})}\right)\right.$ is a vector in $\mathbb{C}^{2 N+1}$ defined by [30]:

$$
\mathcal{F}^{\prime}\left(\mathbf{c}^{(\mathbf{m})}\right)=\mathbf{A}^{*}\left(\mathbf{A} \mathbf{c}^{(\mathbf{m})}-\widetilde{\mathbf{u}}_{\infty}\right)+\mu \mathbf{D}^{*} \Psi^{-1} \mathbf{D} \mathbf{c}^{(\mathbf{m})}
$$

Observe that the Newton algorithm requires to solve -at each iteration- a $(2 N+1) \times$ $(2 N+1)$ linear system which is, in practice, a small system that can be solved with a direct method such as the LU factorization.

\subsection{The Multi-Step Solution Procedure: Algorithm Description}

A preliminary numerical investigation performed by the authors reveals that the Newton algorithm given by equations (9)-(10) delivers a far-field pattern with a high level of accuracy at points close to the given observation angles. However, such an accuracy deteriorates dramatically at points far from the measured data. For this reason, the following three-step procedure for extending the FFP to full aperture is proposed.

Step 1. The given data in this step are the frequency regime $k a$ ( $a$ characterizes the dimension of the scatterer), the truncation order of the Fourier seires (see Eq. (3) is $N$, and the number of FFP measurements $M$. The value of $N$ depends on $k a$. Typically, $N \approx k a$, which means $2 k a+1$ modes are left in the truncated series. The FFP data $\widetilde{\mathbf{u}}_{\infty}=\left[\widetilde{u}_{\infty}\left(\hat{\theta}_{1}\right), \cdots, \widetilde{u}_{\infty}\left(\hat{\theta}_{M}\right)\right]^{T}$ are measured at $M$ points in 
a given observation sector, as depicted in Figure 1 Next, apply a multi-stage strategy to enrich -at each stage- the FFP measurements by only two additional adjacent values. More specifically, do the following:

Step 1.1. Solve the TV-regularized minimization problem 8 using Newton iteration equations (9)-10. Proceed as follows:

- Initialize the Fourier coefficient vector $\mathbf{c}=\mathbf{c}^{(\mathbf{0})}$ and compute the FFP $\mathbf{u}_{\infty}^{(\mathbf{0})}$ corresponding to $\mathbf{c}^{(\mathbf{0})}$.

- For a given $\mu$ and $\beta$, apply the Newton algorithm to the solution of the regularized IFP problem given by Eq. (8) until convergence/stagnation of the residual which is the 2-norm of the relative error on the FFP, i.e.

$$
\frac{\left(\sum_{j=1}^{M}\left|u_{\infty}^{(m)}\left(\hat{\theta}_{j}\right)-\widetilde{u}_{\infty}\left(\hat{\theta}_{j}\right)\right|^{2}\right)^{\frac{1}{2}}}{\left(\sum_{j=1}^{M}\left|\widetilde{u}_{\infty}\left(\hat{\theta}_{j}\right)\right|^{2}\right)^{\frac{1}{2}}}<\epsilon_{1}
$$

where $\mathbf{u}_{\infty}^{(\mathbf{m})}$ is the computed FFP at Newton iteration $m$ and $\epsilon_{1}$ is a prescribed tolerance. $\epsilon_{1}$ is typically the noise level in the original data.

- Compute the new FFP and store its values at $M+2$ observation points. These points are the ones located at the original $M$ measurements and 2 additional points that are adjacent to the initial ones, as depicted in Figure 2(a) Note that the values of the original FFP data are replaced by the computed ones. Hence, at the end of this step, the FFP data are enriched by two new observation points and the original measurements are updated by the computed ones. Also, store the Fourier coefficients vector denoted by $\mathbf{c}^{[1]}=\left[c_{-N}^{[1]}, \cdots, c_{0}^{[1]}, \cdots, c_{N}^{[1]}\right]^{T}$ corresponding to the FFP computed at the last iteration of the Newton algorithm.

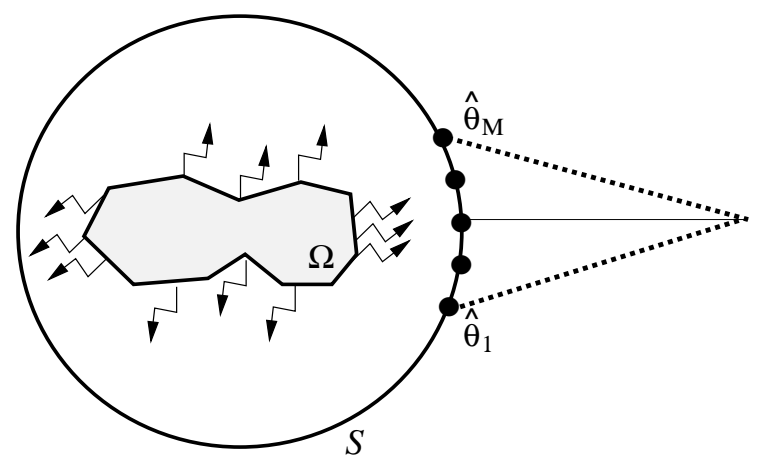

Figure 1: The given (few) FFP measurements for Step 1 are located at points represented by "•". 


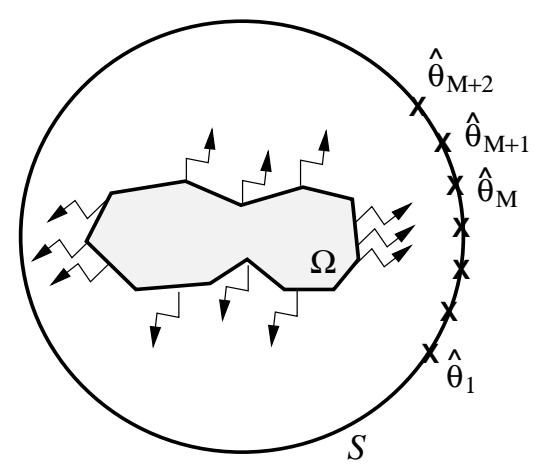

(a) The Given FFP data are extended at the end of Step 1.1 by 2 measurements located at $\hat{\theta}_{1}$ and $\hat{\theta}_{M+2}$.

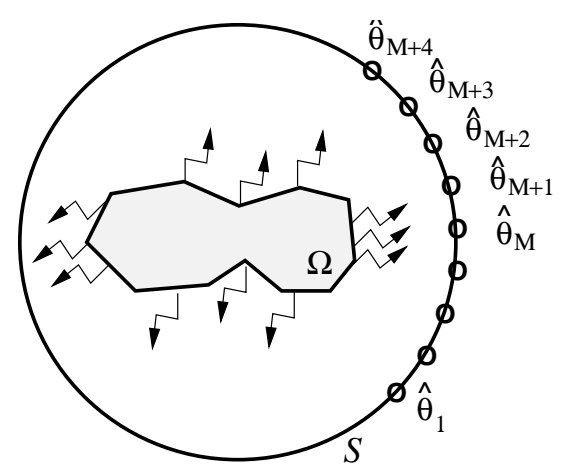

(b) The computed FFP measurements in Fig 2(a) are extended at the end of Step 1.2 by 2 measurements located at $\hat{\theta}_{1}$ and $\hat{\theta}_{M+4}$.

Figure 2: Extension process of the FFP in Step 1.

Step 1.2. Repeat the solution procedure described in Step 1.1 where -this timethe FFP measurements (the reference solution for the Newton algorithm) are the $M+2$ values of the FFP computed and stored in Step 1.1, i.e., $\widetilde{\mathbf{u}}_{\infty}=\left[\widetilde{u}_{\infty}\left(\hat{\theta}_{1}\right), \cdots, \quad \widetilde{u}_{\infty}\left(\hat{\theta}_{M}\right), \widetilde{u}_{\infty}\left(\hat{\theta}_{M+1}\right), \widetilde{u}_{\infty}\left(\hat{\theta}_{M+2}\right)\right]^{T}$. Note that the stopping criterion of the Newton algorithm now is the 2-norm of the residual at the $M+2$ observation points, that is:

$$
\frac{\left(\sum_{j=1}^{M+2}\left|u_{\infty}^{(m)}\left(\hat{\theta}_{j}\right)-\widetilde{u}_{\infty}\left(\hat{\theta}_{j}\right)\right|^{2}\right)^{\frac{1}{2}}}{\left(\sum_{j=1}^{M+2}\left|\widetilde{u}_{\infty}\left(\hat{\theta}_{j}\right)\right|^{2}\right)^{\frac{1}{2}}}<\epsilon_{1}
$$

Then,

- Compute the new FFP and store its values at $M+4$ observation points. These points are the ones located at the $M+2$ previous measurements and 2 additional points that are adjacent to the previous ones, as depicted in Figure 2(b) Hence, the values of the original $M+2$ FFP data are updated by the computed ones. Observe that, at the end of this step, the original $M$ FFP data are updated and enriched by four measurements.

- Store the Fourier coefficients vector denoted by $\mathbf{c}^{[2]}=\left[c_{-N}^{[2]}, \cdots, c_{0}^{[2]}, \cdots, c_{N}^{[2]}\right]^{T}$ corresponding to the FFP computed at the last iteration of the Newton algorithm.

Step 1.3. Repeat this multi-stage process until its stagnation, i.e., the values of the Fourier coefficients vector -stored each time- are no longer changing:

$$
\left\|\mathbf{c}^{[\mathbf{m}+\mathbf{1}]}-\mathbf{c}^{[\mathbf{m}]}\right\|_{2}=\left(\sum_{j=-N}^{N}\left|c_{-N}^{[m+1]}-c_{-N}^{[m]}\right|^{2}\right)^{\frac{1}{2}}<\epsilon_{2}
$$


where $\epsilon_{2}$ is a fixed tolerance. Typically, $\epsilon_{2} \approx 10^{-6}$. At stagnation, compute the new FFP and store its values in aperture of $90^{\circ}$, that is $\widetilde{\mathbf{u}}_{\infty}=$ $\left(\widetilde{u}_{\infty}\left(\hat{\theta}_{1}\right), \cdots, \widetilde{u}_{\infty}\left(\hat{\theta}_{91}\right)\right)$. These values are located in the sector of the original measurements, as depicted in Figure 3(a)

Step 2. Repeat the multi-stage procedure described in Step 1 using this time the following initial data:

- The number of Fourier coefficients is increased by 2, i.e., $N \leftarrow(N+1)$.

- The FFP measurements are the values the computed FFP at the end of Step 1.3. These measurements are given in an aperture of $90^{\circ}$ i.e. $M \leftarrow 91$ (see Figure 3(a).

At stagnation (at the end of Step 2), compute the new FFP and store its values at $91+2 P$ new observation points ( the number of extended FFP measurements is always even). These points are the ones located in the region of the previous $90^{\circ}$ aperture measurements plus $2 P$ additional points that are adjacent to them. Hence, at the end of step 2, the FFP is computed over an aperture of $(90+2 P)^{o}$ (for $P=1$, see Figure 3(b) .

Step 3. The full aperture of the FFP is computed in this step using a Tikhonov regularization technique. The FFP data are the values stored at the end of Step 2, i.e. $\widetilde{\mathbf{u}}_{\infty}=\left[\widetilde{u}_{\infty}\left(\hat{\theta}_{1}\right), \cdots, \widetilde{u}_{\infty}\left(\hat{\theta}_{91+2 P}\right)\right]^{T}$. Proceed as follows:

- First, compute the final values of the Fourier coefficients $\hat{\mathbf{c}}=\left[\hat{c}_{-N-1}, \cdots, \hat{c}_{0}\right.$, $\left.\cdots, \hat{c}_{N+1}\right]^{T}$ by solving the linear system:

$$
\hat{\mathbf{c}}=\left(\mathbf{A}^{*} \mathbf{A}+\mu \mathbf{I}\right)^{-1} \mathbf{A}^{*} \widetilde{\mathbf{u}}_{\infty}
$$

where $\mu$ is the regularization parameter chosen using a trial and error strategy.

- Then, evaluate the full aperture of the FFP using the Fourier series expansion:

$$
\widetilde{u}_{\infty}(\theta)=\sum_{n=-N-1}^{N+1}(-i)^{n} \hat{c}_{n} e^{i n \theta} ; \quad \theta \in[0,2 \pi)
$$

\subsection{Computational Complexity}

The multi-stage methodology outlined previously for extending the FFP data to full aperture requires solving mainly $(2 N+1) \times(2 N+1)$ linear systems which are smallscale systems since $2 N+1$ is the number of Fourier modes in the truncated series. Therefore, these linear system can be easily solved using direct methods such as the standard $L U$ factorization. The number of these linear systems depends however on the number of Newton iterations (typically no more than 10 iterations) and the number of trials -at each Newton iteration- to select the "best" values of the parameters $\mu$ and $\beta$ (about 40 trials). 


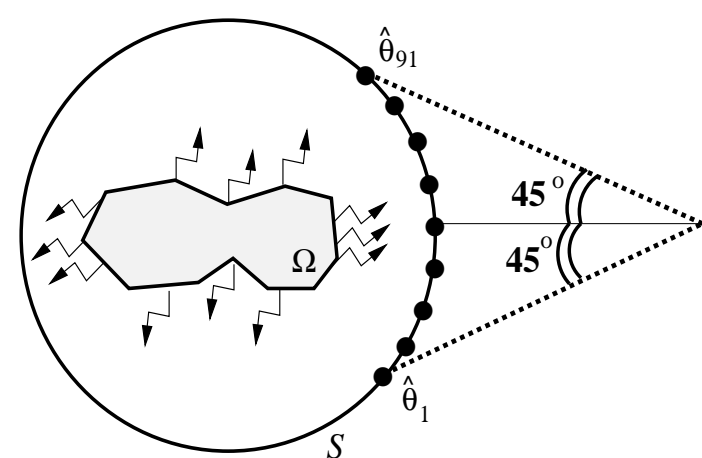

(a) The initial FFP measurements in Step 2 are given in a $90^{\circ}$ aperture. These data are obtained at the end of Step 1.

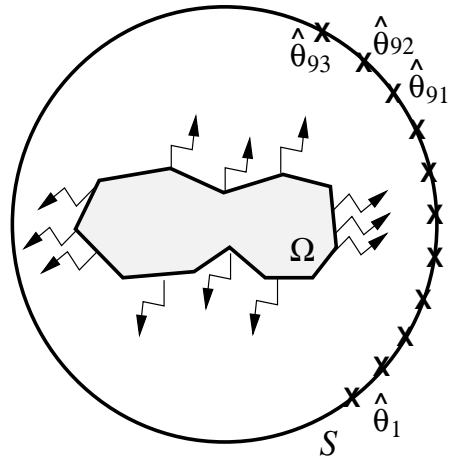

(b) The computed FFP measurements in Fig 3(a) are extended at the end of Step 2.1 by 2 measurements located at $\hat{\theta}_{1}$ and $\hat{\theta}_{93}$.

Figure 3: Extension process of the FFP in Step 2.

\section{Illustrative Numerical Results}

In this section, we illustrate the potential of the proposed solution methodology for enriching FFP data from the knowledge of few observation points: backscattering measurements and a two-degree aperture. For this purpose, we have performed numerical experiments to illustrate the sensitivity of the performance of the proposed methodology to the noise level and the frequency regime. All the numerical experiments were performed using synthetic FFP data corresponding to the acoustic scattered field by a sound-soft disk-shaped scatterer. This synthetic field can be computed analytically using the following Fourier series [2]:

$$
u_{\infty}(\theta)=\sum_{n=-0}^{\infty} \gamma_{n}(-1)^{n} \frac{J_{n}(k a)}{H_{n}^{1}(k a)} \cos (n \theta) \quad ; \quad \theta \in[0,2 \pi)
$$

where $\gamma_{0}=1$ and $\gamma_{n}=2 ; \forall n \geq 1 . a$ is the radius of the considered disk-shaped obstacle. $J_{n}$ (resp. $H_{n}^{1}$ ) is the Bessel function (resp. Hankel function) of first kind of order $n$ [32]. Note that the Dirichlet boundary condition characterizing a sound-soft scatterer is used here only for simplicity and that the results presented herein apply to all types of admissible boundary conditions.

\subsection{FFP Extension from Backscattering Measurements}

We consider the case where the data are measured in a $0^{\circ}$-aperture, i.e. the FFP is measured at one point only $(M=1)$. First, we present numerical results to assess the sensitivity to the noise level of the reconstruction procedure. We consider four level of white noise (the 2-norm sense): noise free $(0 \%)$, low level (1\%), medium level $(5 \%)$, and high level $(10 \%)$. In all the experiments, we have set $k a=1$. The results are reported in Figures (4)-(7) and Table 1] The following observations are noteworthy:

i. In all cases, the proposed procedure is able to reconstruct the FFP. The reconstruction accuracy is much more better over a $90^{\circ}$-aperture, which is of a great 
Table 1: Sensitivity of the 2-norm relative error of the enriched FFP to the noise level. Case of backscattering data $(M=1)$ and $\mathrm{ka}=1$.

\begin{tabular}{|c||c|c|}
\hline Noise level & Extension over $90^{\circ}$ & Extension over $360^{\circ}$ \\
\hline \hline $0 \%$ & $5.1121 \%$ & $10.5880 \%$ \\
$1 \%$ & $6.2285 \%$ & $11.9970 \%$ \\
$5 \%$ & $6.0375 \%$ & $21.3259 \%$ \\
$10 \%$ & $8.2838 \%$ & $24.7173 \%$ \\
\hline
\end{tabular}

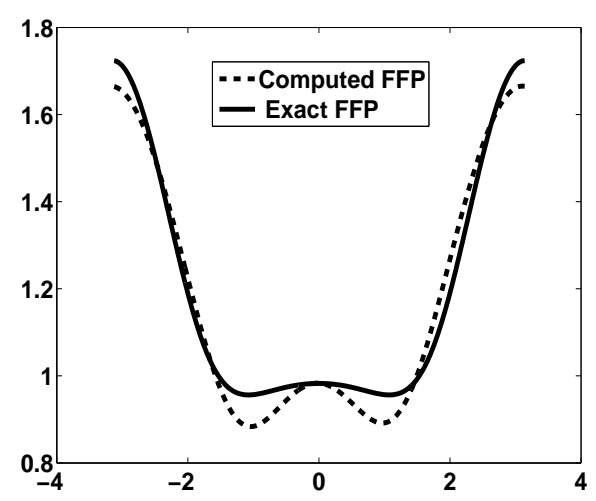

(a) Comparison between absolute values of exact and computed FFP at the end of Step 1 (at stagnation, i.e., $M=8$ ).

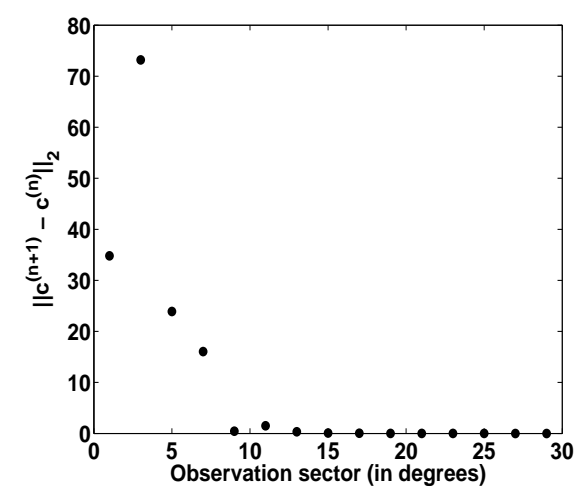

(b) Convergence history of the solution procedure at Step 1 . The algorithm stagnates when $M=8$.

Figure 4: Enrichment of the FFP from a backscattering measurement $(M=1)$ using Step 1 only. Case: $k a=1$ and noise level $0 \%$.

interest when solving IOP problems with regularized Newton-type methods. In- 


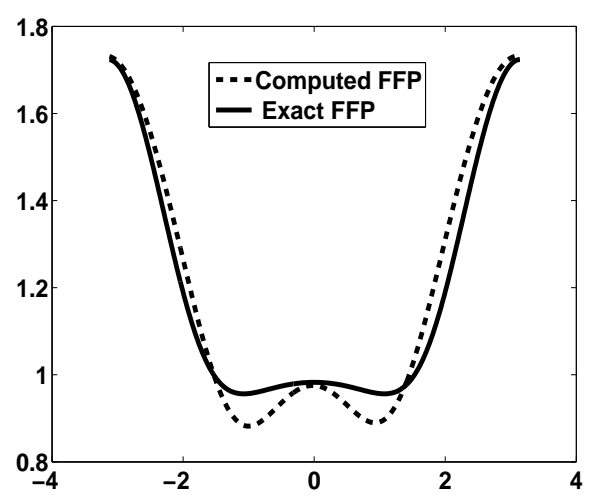

(a) Comparison between absolute values of exact and computed FFP at the end of Step 1 (at stagnation, i.e., $M=8$ ).

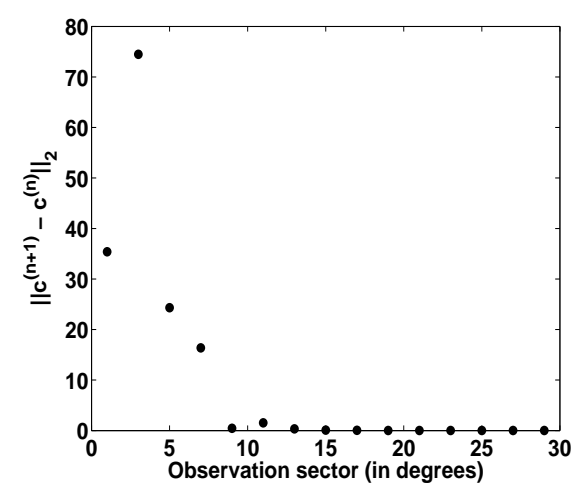

(b) Convergence history of the solution procedure at Step 1. The algorithm stagnates when $M=8$.

Figure 5: Enrichment of the FFP from a backscattering measurement $(M=1)$ using Step 1 only. $k a=1$ and noise level $1 \%$.

deed, as stated earlier, it is possible to succeed in solving IOP problems using regularized iterative-type methods when the FFP data are measured over a $90^{\circ}$ aperture only.

ii. In the case of a noise free backscattering measurement, the reconstruction of the FFP is already pretty good at the end of Step 1 of the procedure (the relative error on the full aperture is about 10\% (see Figure 4 and Table 1. In fact, we have noticed that, in the case of backscattering measurements, as long as the noise level is low (less than 1\%), Step 1 suffices to reconstruct accurately the FFP (see Figure 5 and Table 11. For higher noise level (5\% and 10\%), we have noticed that the procedure at Step 2 does not enrich the $90^{\circ}$-aperture delivered by Step 1 (the algorithm stagnates in Step 2). Therefore, Step 3 uses the computed FFP at Step 1 in the $90^{\circ}$-aperture, and delivers the field depicted in Figures (6)-(7)

iii. As expected, the accuracy of the FFP reconstruction deteriorates with the increase of the noise level. However, such a loss of accuracy is relatively slow, as indicated in Table 1 In addition, the reconstruction of the FFP over a $90^{\circ}-$ 
aperture remains accurate up to the noise level (see Table 1 , which is, as mentioned above, of practical interest. 


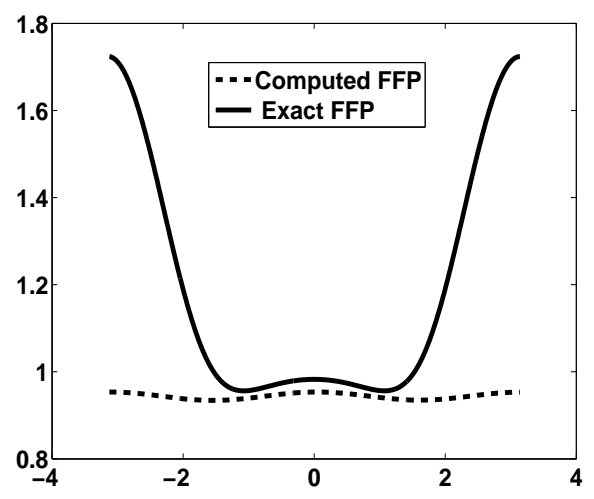

(a) Comparison between absolute values of exact and computed FFP at the end of Step 1 (at stagnation, i.e., $M=12$ ).

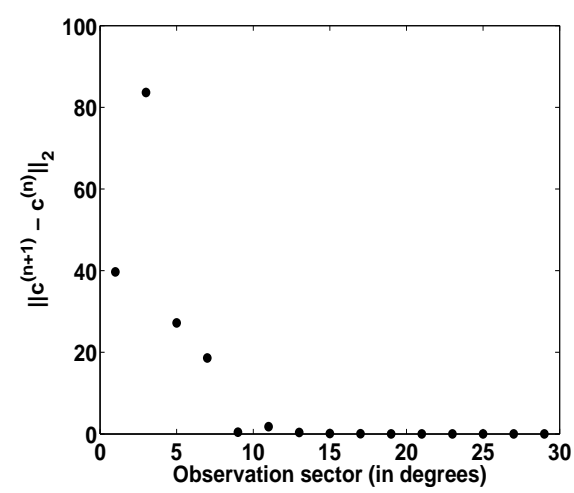

(b) Convergence history of the solution procedure at Step 1. The algorithm stagnates when $M=12$.

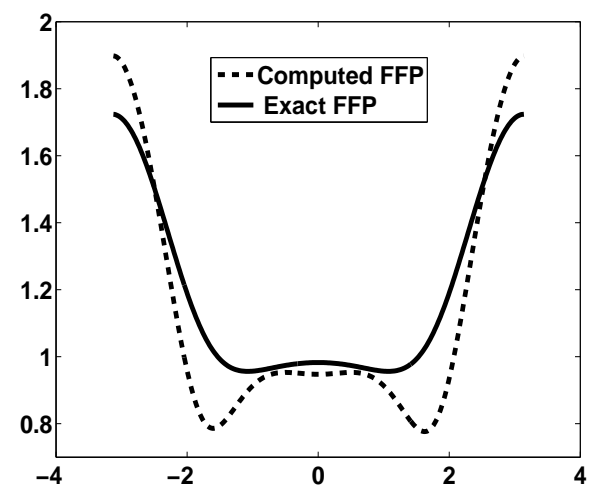

(c) Comparison between absolute values of exact and computed FFP at the end of Step 3. Initial data from Step 1: $\mathrm{M}=90$.

Figure 6: Enrichment of the FFP from a backscattering measurement $(M=1)$. No extension is obtained from Step 2. Case: $k a=1$ and noise level $5 \%$. 


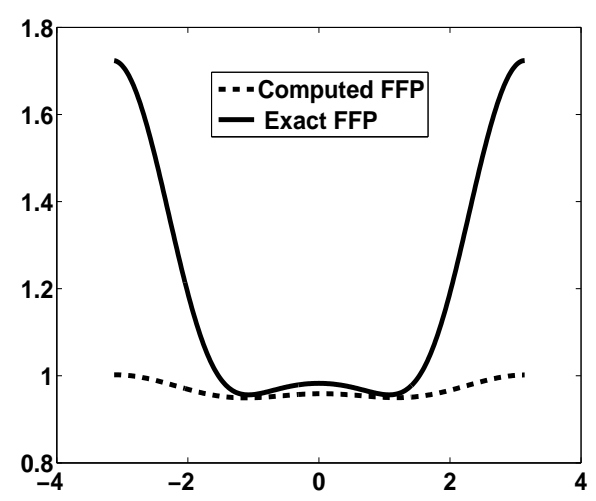

(a) Comparison between absolute values of exact and computed FFP at the end of Step 1 (at stagnation, i.e., $M=12$ ).

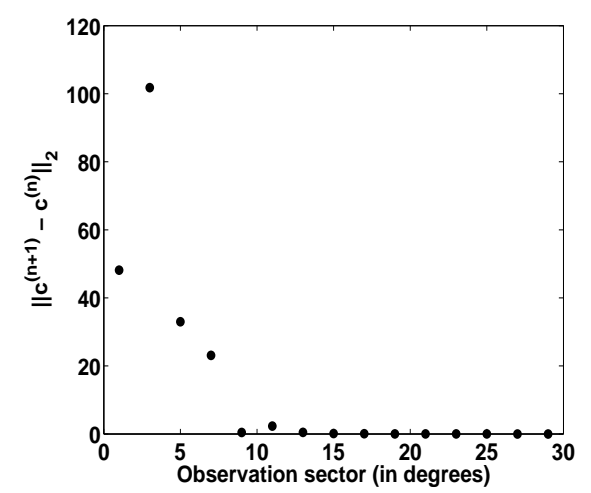

(b) Convergence history of the solution procedure at Step 1 . The algorithm stagnates when $M=12$.

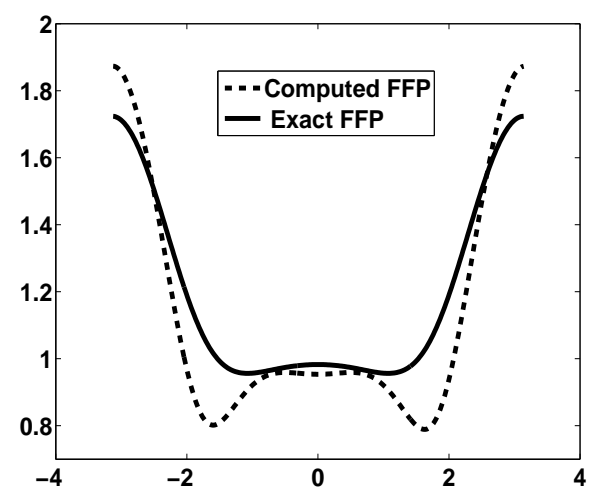

(c) Comparison between absolute values of exact and computed FFP at the end of Step 3. Initial data from Step 1: $\mathrm{M}=90$.

Figure 7: Enrichment of the FFP from a backscattering measurement $(M=1)$. No extension is obtained from Step 2. Case: $k a=1$ and noise level $10 \%$. 
Table 2: Sensitivity of the 2-norm relative error of the enriched FFP to the noise level. Case of a two-degree aperture $(M=3)$ and $\mathrm{ka}=1$

\begin{tabular}{|c||c|c|}
\hline Noise level & Extension over $90^{\circ}$ & Extension over $360^{\circ}$ \\
\hline \hline & & \\
\hline$\%$ & $5.4452 \%$ & $12.5250 \%$ \\
$10 \%$ & $6.2115 \%$ & $11.3946 \%$ \\
& $10.2960 \%$ & $19.3652 \%$ \\
\hline
\end{tabular}

\subsection{FFP Extension from a two-degree aperture}

Next, we analyze the sensitivity of the reconstruction procedure to the size of the FFP data (the number of measurements). We consider the cases of interest, that is, where FFP data are still not enough to solve IOP problems using the existing numerical methods. For illustration purpose, we present the case where the FFP is measured from backscattering in a two-degree aperture, i.e. three measurements are available $(M=3)$. This is a typical (and practical) situation where the enrichment of the data is a prerequisite step for solving successfully IOP problems. Similarly to the backscattering case, we have performed numerical experiments when the data are tainted with three different levels of white noise: low level (1\%), medium level (5\%), and high level (10\%). In all the experiments, we have set $k a=1$. The results are reported in Figures (8)-(10) and Table 2 The following observations are noteworthy:

i. In all cases, the proposed procedure is successful in reconstructing the field over a $90^{\circ}$-aperture, as indicated in Table 2 which is (again) very promising for solving efficiently IOP problems.

ii. Unlike the case of backscattering reconstruction, we have observed that Step 2 does play a role in the reconstruction for data given in a wider aperture, regardless of the noise level as illustrated in Figures [8-10. However, we have observed that the extension of the $90^{\circ}$-aperture at Step 2 does not exceed a sector of $2^{\circ}$. In fact, our numerical investigation reveals that Step 2 does not extend the $90^{\circ}$-aperture more than $6^{\circ}$.

iii. For low noise level (1\%), the accuracy is comparable to the case of the reconstruction from backscattering data (see Tables 112). On the other hand, for medium noise level (5\%), the procedure is sensitive to the size of the data. Indeed, we observe that the relative error on the full reconstructed FFP from a $2^{o}$-aperture is reduced by a factor 2 compared to the reconstruction when starting from a $0^{\circ}$-aperture, as indicated in Table 1 and 2.

iv. For higher noise level, the procedure performs slightly better when the size of the FFP data is increased. Indeed, as illustrated in Tables 12 the accuracy is improved by about $6 \%$ in the case of an extension over a full aperture from a $2^{\circ}$-aperture compared to the reconstruction from $0^{\circ}$-aperture. Applying filtering procedures, as suggested in [15] could be a possible alternative to reduce the 
level of errors on the reconstructed full aperture, and therefore to improve the accuracy. 


\subsection{Sensitivity of the FFP Extension to the Frequency}

We have investigated the performance of the proposed procedure for reconstructing the FFF field in the high frequency regime. We have found the following:

i. The proposed procedure performs poorly in the case of full extension (over $360^{\circ}$ aperture) of the FFP field from few noisy measurements.

ii. The procedure solution methodology remains efficient for partial enrichment of the FFP field using Step 1 only. More specifically, at the end (stagnation) of Step 1, the procedure delivers the FFP field over a $90^{\circ}$-aperture with a good level of accuracy, as illustrated in Table 3 The results reported in Table 3 are the relative errors of the FFP extension over a $90^{\circ}$-aperture from backscattering (resp. twodegree) measurements corresponding to five frequencies: $k a=1,2,3,4$ and 5 . The original FFP measurements in these experiments were contaminated with $5 \%$ of white noise. The following observation are noteworthy.

- As clearly illustrated in Table 3 the level of accuracy in the extension remains acceptable (given the level of noise in the data) for frequencies values $k a \leq 4$. This observation is very important to regularized iterative solvers that require data to be given in -at least- a $90^{\circ}$-aperture for solving successfully IOP problems.

- As expected, the extension of the FFP field from a two-degree aperture measurements is more accurate than from backscattering measurements.

- The level accuracy in the extension deteriorates dramatically for high values of the frequency $(k a \geq 5)$. Yet, the reconstructed field for such frequencies can be incorporated in an IOP iterative solver after reducing -if possible- the level of errors by applying a noise filtering-type procedure [15].

Table 3: Effect of the frequency on the 2-norm relative error of the FFP extension over a $90^{\circ}$-aperture. Original measurements tainted with $5 \%$ of white noise.

\begin{tabular}{|c||c|c|}
\hline Frequency $k a$ & $\begin{array}{c}\text { Extension from } \\
\text { Backscattering Measurement } \\
M=1\end{array}$ & $\begin{array}{c}\text { Extension from } \\
\text { a Two-Degree Aperture } \\
M=3\end{array}$ \\
\hline \hline & & \\
\hline & & \\
2 & $4.5782 \%$ & $3.6299 \%$ \\
3 & $15.0690 \%$ & $9.8891 \%$ \\
4 & $20.7420 \%$ & $11.2520 \%$ \\
5 & $20.8970 \%$ & $21.6600 \%$ \\
& $32.4070 \%$ & $31.0140 \%$ \\
\hline
\end{tabular}




\section{Summary and Conclusion}

In response to the pressure need for FFP data in large apertures $\left(90^{\circ}\right.$-apertures at least) for solving efficiently inverse obstacle problems with iterative solution methodologies, we have designed a procedure that extends few noisy FFP measurements to larger apertures. The proposed method is a three-stage procedure where the total variation of the FFP field is used for regularization. Using synthetic FFP data, we have perofrmed numerical experiments to assess the performance of the proposed method. The numerical results indicate that the procedure extends successfully noisy backscattering measurements to full aperure $\left(360^{\circ}\right.$-aperture). Such an efficiency is achieved even in the presence of high level of noise, but for measured data corresponding to relatively small frequency values. Note that in the particular case of backscattering measurements and low noise level (less than 1\%), the field is reconstructed accurately over the full aperture at the end of Step 1 of the proposed procedure. For higher level of noise, the three steps of the procedure are crucial for a successful extension. For relatively high frequency values, the procedure performs poorly for full aperture extension.

However, the proposed procedure remains efficient for extending the FFP field over a $90^{\circ}$-aperture after applying Step 1 only.

\section{References}

[1] C. R. Vogel, Computational Methods for inverse Problems, Frontiers in Applied Mathematics, Society for Industrial and Applied Mathematics (SIAM), Philadelphia, PA, 2002.

[2] D. Colton and R. Kress, Inverse acoustic and electromagnetic scattering theory, Applied Mathematical Sciences 93, Springer-Verlag, 1992.

[3] J. Hadamard, Lectures on Cauchy's problem in linear partial differential equations, Yale University Press, 1923.

[4] R. Djellouli, Inverse Acoustic Problems, In: Computational Methods for Acoustics Problems, (F. Magoulès, editor), Saxe-Coburg Publications, (2008), pp. 263294.

[5] C. Farhat, R. Tezaur, R. Djellouli, On the solution of three-dimensional inverse obstacle acoustic scattering problems by a regularized Newton method, Inverse Problems, 18, (2002), pp. 1229-1246.

[6] R. L. Ochs, The limited aperture problem of inverse acoustic scattering: Dirichlet boundary conditions, SIAM J. Appl. Math. 47, (1987), pp. 1320-1341.

[7] R. M. Lewis, Physical optics inverse diffraction, IEEE Trans. Antennas and Propagation, 17, (1969), pp. 308-314.

[8] R. D. Mager, N. Bleistein, An approach to the limited aperture problem of physical optics far-field inverse scattering, Tech. Report MS-R-7704, University of Colorado at Denver, 1977.

[9] N. Bleistein, J. K. Cohen, A survey of recent progress on inverse problems, Tech. Report MS-R-7806, University of Colorado at Denver, 1977. 
[10] A. Zinn, On an optimization method for full- and limited-aperture problem, In: inverse acoustic scattering for a sound-soft obstacle, Inverse Problems, 5, (1989), pp. 239-253.

[11] R. Kress, Integral equations methods in inverse acoustic and electromagnetic scattering, In: Boundary Integral Formulations for Inverse Analysis, (Ingham and Wrobel, eds.), Computational Mechanics Publications, Southampton, (1997), pp. $67-92$.

[12] R. Kress, W. Rundell, Inverse obstacle scattering using reduced data, SIAM J. Appl. Math., 59, (1999) pp. 442-454.

[13] F. Oukaci, Quelques problèmes numériques d'identification de forme en diffraction acoustique, Ph. D. Thesis, Université de Technologie de Compiegne, 1999.

[14] R. Djellouli, R. Tezaur, and C. Farhat, On the solution of inverse obstacle acoustic scattering problems with a limited aperture, in: Mathematical and Numerical Aspects of Wave Propagation (Cohen et al. eds.), Jyväskylä, (2003), pp. 625-630.

[15] Y. Olshansky, I. Stainvas, and E. Turkel, Simultaneous Scatterer Shape Estimation and Far-Field Pattern Denoising, in: Proceedings of the ninth International Conference on Mathematical and Numerical Aspects of Wave Propagation (Barucq et al. eds.), Pau, (2009), pp. 306-307.

[16] C. H. Wilcox, Scattering theory for the D'Alembert equation in exterior domains, Springer-Verlag, Lecture Notes in Mathematics, 442, 1975.

[17] A. G. Ramm, Scattering by obstacles, Reidel, Dotrecht, 1986.

[18] P. D. Lax and R. S. Philips, Scattering theory, Academic Press, New York, 1967.

[19] J. Sanchez Hubert and E. Sanchez-Palencia, Vibration and Coupling of Continuous Systems, Springer, Berlin, 1989.

[20] F. Magoulès, Computational Methods for Acoustics Problems, Saxe-Coburg Publications, 2008.

[21] A. Kirsch, R. Kress, Uniqueness in inverse obstacle scattering, Inverse Problems, 9, (1993), pp. 285-299.

[22] A. B. Bakushinsky and M. Yu. Kokurin, Iterative methods for Approximate Solution of Inverse Problems, Mathematics and its Applications, 577, Springer, 2004.

[23] A. N. Tikhonov, V. Y. Arsenin, Solutions of ill-posed problems, Winston and Sons, Washington, 1977.

[24] [43] V. A. Morozov, On the solution of functional equations by the method of the regularization, Doklady, 167, (1966), pp. 414-417.

[25] A. Kirsch, An introduction to the mathematical theory of inverse problems, Springer-Verlag, New-York, 1996.

[26] C. R. Vogel, Total Variation regularization for ill-posed problems, Department of Mathematical Sciences Technical Report (1993), Montana State University. 
[27] L. I. Rudin, S. Osher and E. Fatemi, Nonlinear total variation based noise removal algorithms, Proceeding of the 11th Annual International Conference of the center for Nonlinear Studies, Physica D, Vol.60 (1992), pp. 259-268.

[28] R. Acar and C. R. Vogel, Analysis for Bounded Variation Penalty Methods for Ill-Posed Problems, Inverse Problems, Vol. 10, No. 6 (1994), pp.1217-1229.

[29] C. Hansen, Regularization Tools: a Matlab Package for Analysis and Solution of Ill-Posed Problems, Numerical Algorithms 6 (1994), pp. 1-35.

[30] J. Pedersen, Modular Algorithms for Large-Scale Total Variation Image Deblurring, Master Thesis, Technical University of Denmark, 2005.

[31] J.J. Bowman, T.B.A. Senior, P.L.E. Uslenghi, Electromagnetic and acoustic scattering by simple shapes, North-Holland Publishing company, Amsterdam, 1969.

[32] M. Abramovitz, I. Stegun, Handbook of Mathematical Functions with Formulas, Graphs and Mathematical Tables, Dover Publications, New York, 1972. 


\section{Appendix A}

We present in this section auxiliary properties of the total variation (TV) formulation in the simple case of one-dimensional functions. These elementary properties are included only for illustration. For completeness, more results can be found in [26][30], among other references.

Let $f(t): I \subseteq \mathbb{R} \longrightarrow \mathbb{R}$ be a regular function (for example, continuously differentiable) on an interval $I$. Then, the total variation of $f$, denoted by $\mathcal{J}_{T V}(f)$, is given by:

$$
\mathcal{J}_{T V}(f)=\int_{I}\left|f^{\prime}(t)\right| d t=\left\|f^{\prime}(t)\right\|_{1}
$$

Observe that $\mathcal{J}_{T V}(f)$ is the integral of the "variations" $f^{\prime}$ of the function $f$ over the entire domain $I$. For this reason, $\mathcal{J}_{T V}(f)$ is called the total variation of $f$.

Next, we illustrate the effect of the total variation functional when employed as a Tikhonov-like regularization term (penalty-type term). This is achieved by comparing the $L^{1}$ penalty term $\left\|f^{\prime}\right\|_{1}$ introduced by $\mathcal{J}_{T V}(f)$ to the classical $L^{2}$ penalty term $\left\|f^{\prime}\right\|_{2}$ (standard Tikhonov regularization).

Consider the function $f$ depicted in the figure below. It is easy to verify that $f^{\prime}(t)=0$ if $t>l$ and $f^{\prime}(t)=\frac{d}{l}$ if $0<t<l$. In addition, we have:

$$
\left\|f^{\prime}\right\|_{1}=d \quad \text { and } \quad\left\|f^{\prime}\right\|_{2}=\frac{d}{\sqrt{l}}
$$

Eq. 116 reveals the following two features:

i. When using the $L^{1}$-norm, a constant penalty is associated with the variations of $f$, since $\left\|f^{\prime}\right\|_{1}=d$. However, with the $L^{2}$-norm, the penalty associated with the variations of $f$ is inversely proportional to $\sqrt{l}$, hence rapid variations of $f$ are more penalized.

ii. The semi-norm $L^{2}$ admits a unique minimizer (i.e. as $l$ goes to infinity), whereas $L^{1}$ is minimized by an infinite number of functions, which could be a great advantage since the function of interest (or a close approximation of it) could be among these functions.

As stated in the introduction of this paper, because of the non differentiability of the operator $\mathcal{J}_{T V}$, the TV formulation has not been used by applied mathematicians and engineers as a penalty term whenever numerical methods that require the computation of the jacobians are employed. This difficulty can be alleviated by perturbing the operator $\mathcal{J}_{T V}$ [30]. More specifically, we replace the operator $\mathcal{J}_{T V}$ by $\mathcal{J}_{T V, \beta}$ given by:

$$
\mathcal{J}_{T V, \beta}(f)=\int_{I} \sqrt{\left|f^{\prime}(t)\right|^{2}+\beta^{2}} d t
$$

where the positive real number $\beta$ is introduced as a regularity coefficient. Observe that, for a fixed function $f$, we have:

$$
\mathcal{J}_{T V, \beta}(f) \longrightarrow \mathcal{J}_{T V}(f) \quad \text { as } \quad \beta \longrightarrow 0^{+}
$$


Last, the computation of $\mathcal{J}_{T V, \beta}(f)$ requires the evaluation of the derivative of $f$. Unfortunately, it is not possible or easy to evaluate the derivative $f^{\prime}$ for most practical inverse problems. For such cases, one can use finite difference schemes to approximate the derivative of $f$. For example, the use of the central discrete scheme leads to the following algebraic expression approximating the perturbed TV operator $\mathcal{J}_{T V}, \beta$ :

$$
\mathcal{J}_{T V, \beta}(f) \approx J_{T V, \beta}(f)=\left\|\left((\mathbf{D f})^{\mathbf{2}}+\beta^{2} \widetilde{\mathbf{e}}\right)^{\mathbf{1 / 2}}\right\|_{1}
$$

with

$$
\mathbf{D}=\frac{1}{2 h}\left[\begin{array}{cccccc}
-2 & 2 & 0 & 0 & \cdots & 0 \\
-1 & 0 & 1 & 0 & \cdots & 0 \\
\vdots & \vdots & \vdots & \vdots & \vdots & \vdots \\
0 & \cdots & 0 & -1 & 0 & 1 \\
0 & \cdots & 0 & 0 & -2 & 2
\end{array}\right]
$$

Here, $\mathbf{D}$ is the central discrete first derivative operator, used in order to eliminate highly oscillating regularized solutions. $f$ is assumed to vary only in an interval containing the uniformly spread points $t_{1}<\cdots<t_{n}$, with $t_{i+1}-t_{i}=h$, and $\widetilde{\mathbf{e}}$ is a vector of ones. In the Tikhonov setting, there is no need for computing $h$, since it is absorbed into the regularization coefficient and the regularity coefficient $\beta$. Finally, the bold face exponents in Eq. 18) are pointwise operations.

\section{Contents}

\begin{tabular}{lll}
\hline & Introduction & 3
\end{tabular}

2 Preliminaries 4

2.1 The Direct Acoustic Scattering Problem . . . . . . . . . . . . . 5

2.2 The Inverse Obstacle Problem . . . . . . . . . . . . . . . . 5

3 The Multi-Step Procedure for Enriching the FFP data 6

3.1 Problem Statement . . . . . . . . . . . . . . . . . 6

3.2 The total variation regularization technique: A modified procedure . . 7

3.3 The Newton Algorithm . . . . . . . . . . . . . . . . . . 9

3.4 The Multi-Step Solution Procedure: Algorithm Description . . . . . . 9

3.5 Computational Complexity . . . . . . . . . . . . . . . . 12

4 Illustrative Numerical Results 13

4.1 FFP Extension from Backscattering Measurements . . . . . . . . . . 13

4.2 FFP Extension from a two-degree aperture . . . . . . . . . . . . . . 19

4.3 Sensitivity of the FFP Extension to the Frequency . . . . . . . . . 21

5 Summarv and Conclusion 22 


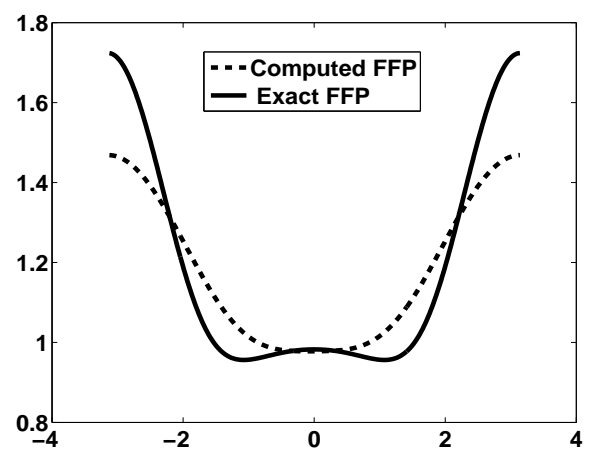

(a) Comparison between absolute values of exact and computed FFP at the end of Step 1 (at stagnation, i.e., $M=16$ ).

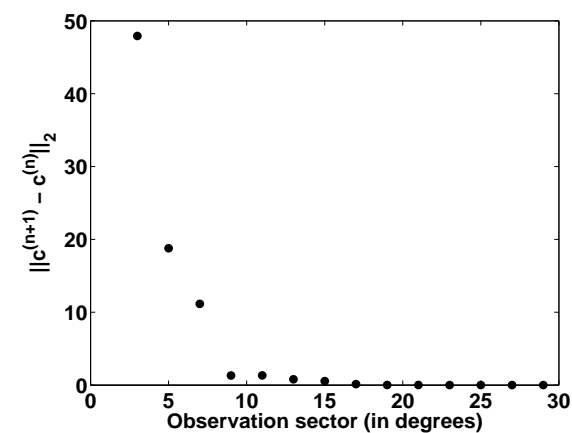

(b) Convergence history of the solution procedure at Step 1. The algorithm stagnates when $M=16$.

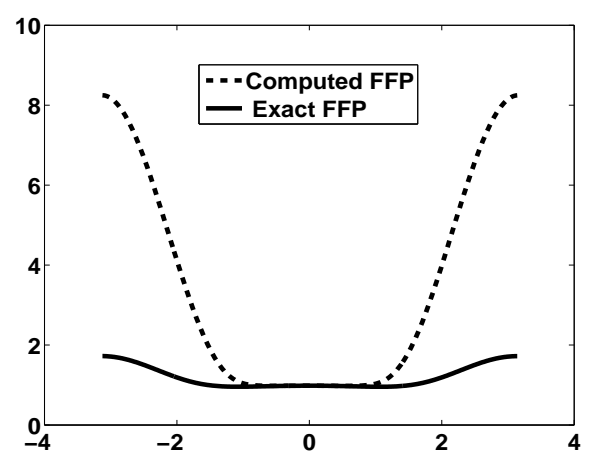

(c) Comparison between absolute values of exact and computed FFP at the end of Step 2 (at stagnation, i.e., $M=92$ ).

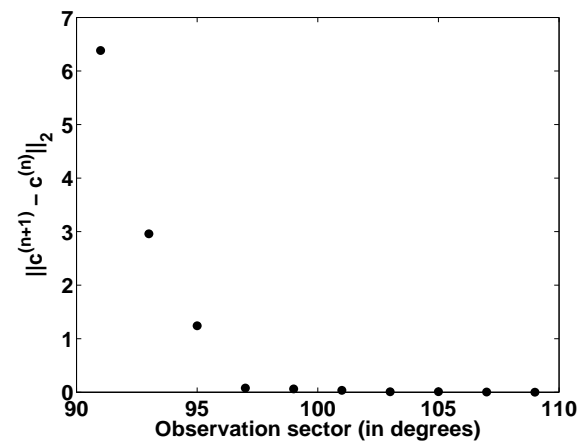

(d) Convergence history of the solution procedure at Step 2. The algorithm stagnates when $M=92$.

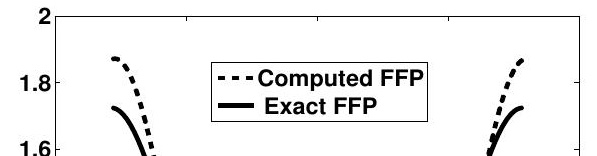




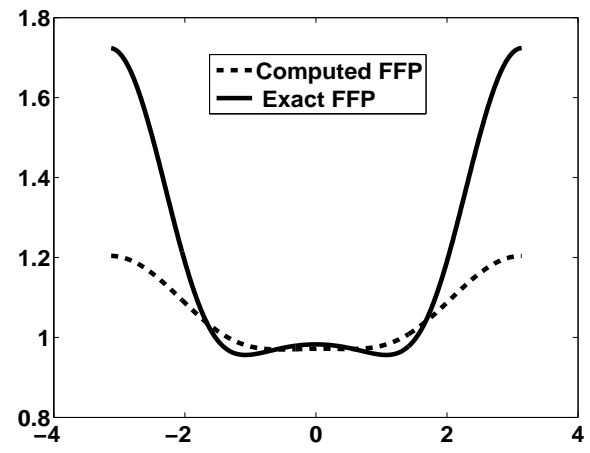

(a) Comparison between absolute values of exact and computed FFP at the end of Step 1 (at stagnation, i.e., $M=16$ ).

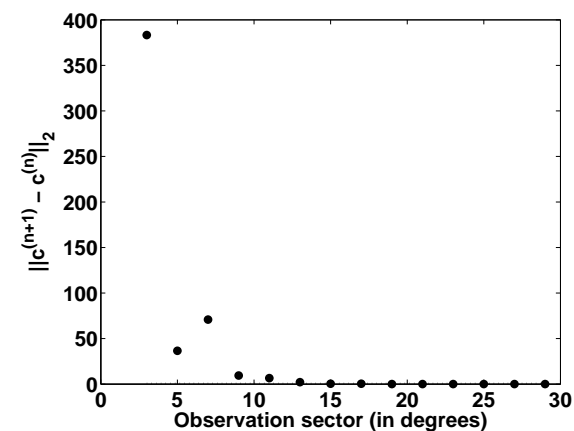

(b) Convergence history of the solution procedure at Step 1 . The algorithm stagnates when $M=16$.

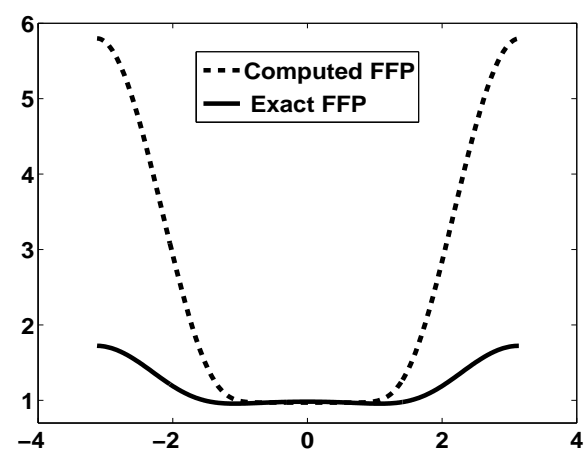

(c) Comparison between absolute values of exact and computed FFP at the end of Step 2 (at stagnation, i.e., $M=92$ ).

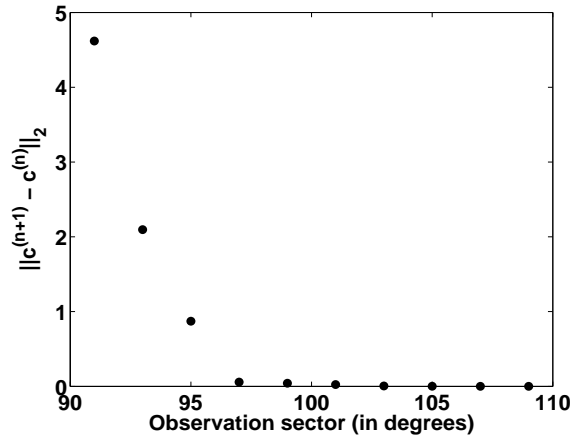

(d) Convergence history of the solution procedure at

Step 2. The algorithm stagnates when $M=92$.

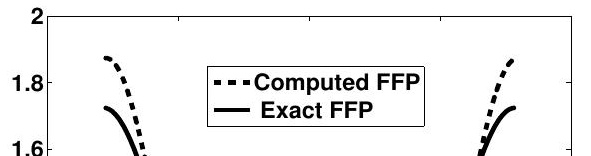




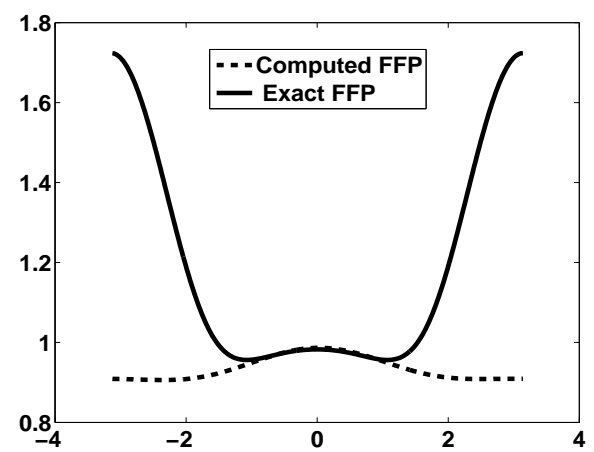

(a) Comparison between absolute values of exact and computed FFP at the end of Step 1 (at stagnation, i.e., $M=16$ ).

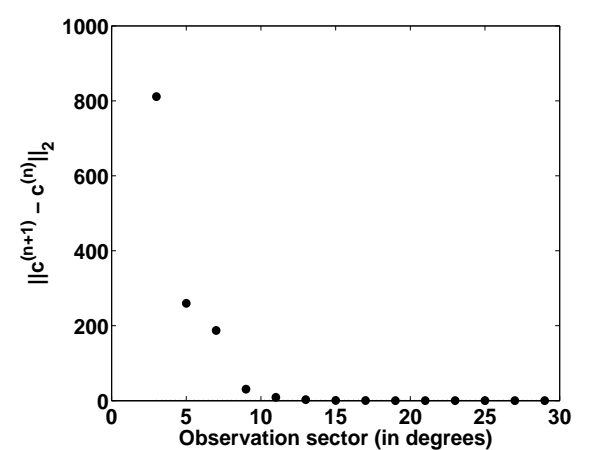

(b) Convergence history of the solution procedure at Step 1. The algorithm stagnates when $M=16$.

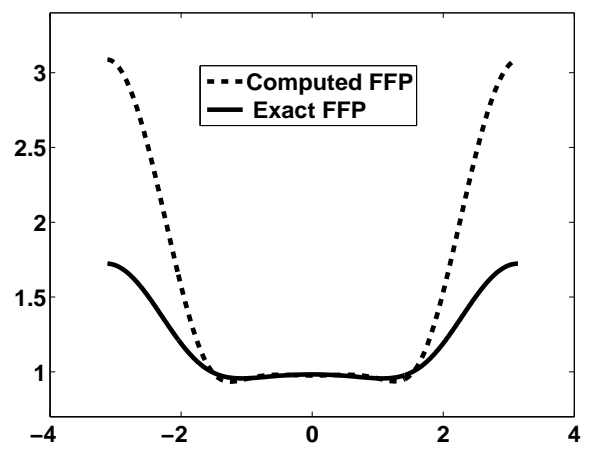

(c) Comparison between absolute values of exact and computed FFP at the end of Step 2 (at stagnation, i.e., $M=92$ ).

RR n 7048

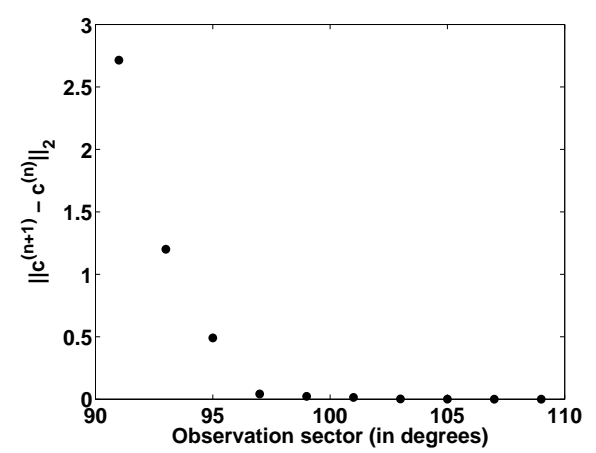

(d) Convergence history of the solution procedure at Step 2. The algorithm stagnates when $M=92$.

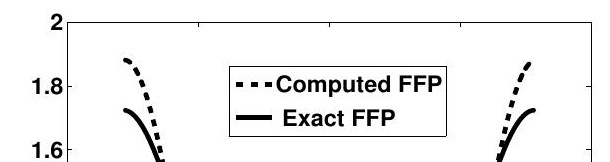




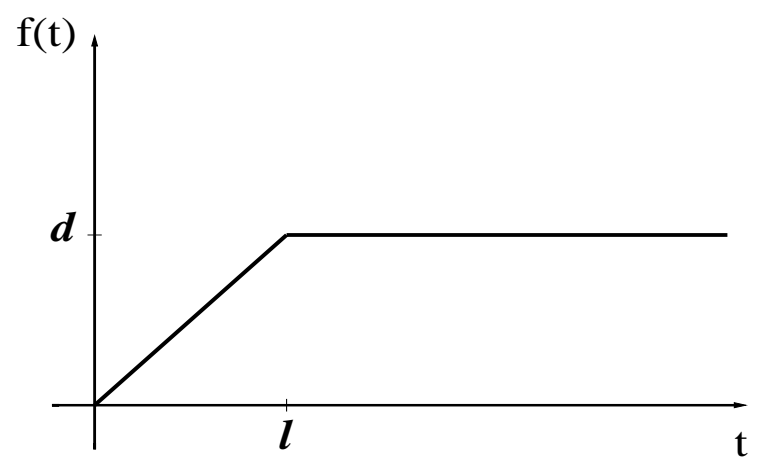


Centre de recherche INRIA Bordeaux - Sud Ouest Domaine Universitaire - 351, cours de la Libération - 33405 Talence Cedex (France)

Centre de recherche INRIA Grenoble - Rhône-Alpes : 655, avenue de l'Europe - 38334 Montbonnot Saint-Ismier Centre de recherche INRIA Lille - Nord Europe : Parc Scientifique de la Haute Borne - 40, avenue Halley - 59650 Villeneuve d'Ascq Centre de recherche INRIA Nancy - Grand Est : LORIA, Technopôle de Nancy-Brabois - Campus scientifique 615, rue du Jardin Botanique - BP 101 - 54602 Villers-lès-Nancy Cedex

Centre de recherche INRIA Paris - Rocquencourt : Domaine de Voluceau - Rocquencourt - BP 105 - 78153 Le Chesnay Cedex Centre de recherche INRIA Rennes - Bretagne Atlantique : IRISA, Campus universitaire de Beaulieu - 35042 Rennes Cedex Centre de recherche INRIA Saclay - Île-de-France : Parc Orsay Université - ZAC des Vignes : 4, rue Jacques Monod - 91893 Orsay Cedex Centre de recherche INRIA Sophia Antipolis - Méditerranée : 2004, route des Lucioles - BP 93 - 06902 Sophia Antipolis Cedex 